\title{
Introduction à la collection «Guides Scientifiques et Techniques du CEFRACOR »
}

Le CEFRACOR (Centre Français de l'Anticorrosion), association à but non lucratif régie par la loi de 1901, fédère 750 ingénieurs, chercheurs et enseignants concernés par les domaines suivants :

- l'étude des différentes formes de corrosion des matériaux,

- les méthodes de protection contre ces dégradations, qui utilisent notamment les traitements chimiques des milieux corrosifs, les revêtements divers et les protections électrochimiques,

- les méthodes de contrôle et de surveillance de leur efficacité.

Ces personnes représentent aussi bien les grands groupes industriels, des PME ou des TPE que les principales universités ou écoles et les centres de recherche spécialisés, ou encore interviennent comme spécialistes consultants.

Le CEFRACOR est partenaire de nombreuses associations et fédérations agissant dans la lutte contre la corrosion. Il est notamment un membre actif de la Fédération Européenne de la Corrosion (EFC), de la Fédération Française pour les sciences de la Chimie (FFC), de la Fédération Française des Matériaux (FFM) et de l'Association pour la Certification et la Qualification des Peintures Anticorrosion (ACQPA). Il est membre de l'AFNOR, étant particulièrement actif dans sa Commission de Normalisation A05AG «Corrosion et protection des matériaux métalliques - Revêtements métalliques et inorganiques ».

Son département CFPC (Conseil Français de la Protection Cathodique) est Organisme de Certification des personnes actives en protection cathodique sous la marque CEFRACOR CERTIFICATION / Protection Cathodique en application des normes NF EN ISO/CEI 17024 et NF EN 15257, son Accréditation ayant été prononcée par le COFRAC à compter du $1^{\text {er }}$ février 2014 pour les secteurs d'application Terre et Mer.

Chacune dans leur domaine, les nombreuses Commissions d'études du CEFRACOR agissent pour le transfert des connaissances et le rapprochement des spécialistes, contribuent aux actions normatives, organisent des manifestations de type colloques, journées d'études ou rencontres, et élaborent des documents scientifiques et techniques. Ces documents peuvent prendre la forme de recommandations diffusées gratuitement sur le site www.cefracor.org, telles les « Recommandations PCRA » qui sont à la disposition des spécialistes pour compléter les normes, standards et recommandations professionnelles dans le domaine de la protection cathodique et des revêtements associés. 
Lorsque les travaux des Commissions d'études conduisent à des documents plus conséquents, ils sont édités sous différentes formes. Les Cahiers du CEFRACOR ont constitué pendant longtemps le moyen privilégié de diffusion et certains sont encore en vente sur le site www.cefracor.org.

Dans le prolongement d'un partenariat établi depuis plusieurs années, notamment autour de la revue Matériaux et Techniques (rendue disponible pour les adhérents du CEFRACOR dans l'Espace Adhérents du site www.cefracor.org), il a été décidé de confier l'édition de Guides Scientifiques et Techniques du CEFRACOR aux Éditions EDP Sciences.

Les deux premiers documents de cette série sont les Guides techniques Revêtements externes des canalisations enterrées ou immergées et des ouvrages connexes et Monitoring de la corrosion interne dans les industries pétrolières et gazières édités en 2016. Ils seront suivis d'autres Guides Scientifiques et Techniques du CEFRACOR dans les prochaines années, notamment sur les thèmes Prédiction et prévision de la corrosion interne dans les industries pétrolières et gazières et Traitements des circuits de refroidissement semi-ouverts.

Marcel Roche

Président du CEFRACOR 